\title{
Confidence Interval for Multivariate Process Capability indices in Statistical Inventory Control
}

\author{
Mustafid $^{1}$, Dwi Ispriyanti ${ }^{2}$, Sugito $^{3}$, Diah Safitri $^{4}$ \\ \{mustafid55@gmail.com,dwiispriyanti@yahoo.com, sugitostat@gmail.com, \\ diahsafitri.fifi@gmail.com \\ ${ }^{1,2,3,4}$ Department of Statistics, Diponegoro University, Semarang, 50275, Indonesia
}

\begin{abstract}
Multivariate process capability indices (MPCI) has important role in the analysis of statistical inventory control determined by several consumer demand as quality characteristics that are correlated. In the inventory control management is also needed confidence interval for MPCI to overcome the uncertain from consumer demand. The research aims to apply the confidence interval for MPCI in statistical inventory control. The case studies conducted on the apparel industry to implement the confidence interval for the MPCI using several types of apparel which is used as the quality characteristics. The upper and lower limits for the intervals from the MPCI are obtained using sample data assuming multivariate normal distribution and stable. Process sample data in stable conditions are obtained by using analysis of multivariate control diagram designed by $\mathrm{T}^{2}$ Hotelling. The MPCI confidence interval can be used as the indicator in determining the number of products provided in inventory based on the number of consumer demand.
\end{abstract}

Keywords: confidence interval, inventory control, multivariate capability indices.

\section{Introduction}

Multivariate process capability index (MPCI) is required for the analysis of process capability using many correlated variables [1]. MPCI is used to make a general measure of process capability in the case of the use of target values and process standard deviations [2]. The main factor in using MPCI is the specification limits for each quality characteristic that represents outcome measures from a process. These specification limits are standard management required by industry or manufacturing, which is the maximum and minimum standard values as a reference, which are referred to as the lower and upper specification limits. In the case of multivariate specification limits represent the area of results received and become more complex than univariate cases [1].

In the industrial and management sectors, MPCI serves as a measure for performance analysis in the field of inventory control. In the apparel industries, apparel products consist of several types of apparel must be produced together, also distributed together to consumers. The role of multivariate control is very important, one of them is for analysis performance of industrial processes by involving several quality characteristics that are correlated with each other.

The measure of process capability is used as a method needed to understand the capabilities of an industrial process according to customer demand. The basic principle in 
process capability analysis is to compare the conditions of customer demand with what must be done by the process. Visually, capability analysis can be done by looking at the plot of process data (such as control chart) and comparing it with the upper specification limits and lower specification limits [3]. If the performance of the process is outside the specification limits, then it is called a process in an unstable condition, which means there is a risk to produce the product as expected.

In many cases, the products produced are explained by more than one characteristic. In the apparel industry, the types of apparel are produced using several different characteristics according to the demands of consumers. Each quality characteristic of the type of apparel product must meet certain specifications $[4,5]$. So that product quality must be assessed on a combined or multivariate characteristic, not only on the value of its individual characteristics.

In the business world, the characteristic of consumer demands always change, likewise MPCI always change at any time [6]. Therefore, it is necessary to know the tolerance limits of MPCI in the form of a confidence interval. The purpose of the research is to apply the confidence interval for MPCI in statistical inventory control with using the case studies for the apparel industry.

\section{Literature review}

\subsection{Process capability indices}

In the industry or management sectors, process capability index $\mathrm{C}_{\mathrm{p}}$ is defined by the ratio of customer demand and proses performance:

$C p=\frac{\text { customers demand }}{\text { process performance }}$

If $\mathrm{C}_{\mathrm{p}}=1$ means the customer response and process performance are the same. In the field of quality management, it is often used specification limits using $3 \sigma$. In this case, for a normally distributed process, then with a probability of $99.73 \%$ the performance of the process will be within $\pm 3 \sigma$. Therefore, the probability for non-conformance or process performance outside the specification limits is $1-0.9973=0.0027$. Using the specification limit, the capability index $C p$ is determined by formula:

$C_{p}=\frac{U S L-L S L}{6 \sigma}$

where LSL and USL are the lower and upper specification limit, and $\sigma$ is the standard deviation of the process [3]. The capability index calculation using formula (1) only uses process variations and compares with the process spread, and do not consider the location or proximity to the target. For calculations capability index using target $\mathrm{T}$, capability index $\mathrm{C}_{p m}$ is determined by formula:

$C_{p m}=\frac{C_{p}}{\sqrt{1+\left(\frac{\mu-T}{\sigma}\right)^{2}}}$

where $\mu$ is the mean and $\sigma$ is the standard deviation of the process [7].

The formulas (1) and (2) are used to analyze capability indices using a single variable or a single quality characteristic. Furthermore, the capability indices for individual quality 
characteristics can be developed to MPCI $[2,8,9,10]$. The capability measures can be used in industry or management to measure process performance and outcome performance according to product or service qualifications. In today's industry, suppliers are asked to provide customers with a measure of product processing capabilities. The process capability index $C_{p}$ and $C_{p m}$ are also used as a benchmark for measuring success in quality improvement projects in accordance with consumer demands.

\subsection{Multivariate process capability indices}

Let $\mathbf{X}=\left(\mathbf{X}_{1}, \mathbf{X}_{2}, \ldots, \mathbf{X}_{\mathrm{q}}\right)^{\prime}$ is q-dimensional variables have a multivariate normal distribution and stable condition in statistical control charts. Let $\mathbf{S}$ is sample variancecovariance matrix. For the purposes of analysis of MPCI, let each quality characteristic of univariate $\mathbf{X}_{\mathrm{j}}$ have a given specification limits $\left(\mathrm{LSL}_{\mathrm{j}}, \mathrm{USL}_{\mathrm{j}}\right), \mathrm{j}=1,2, \ldots \mathrm{q}$. The MPCI is defined by:

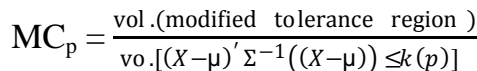

$$
\begin{aligned}
& =\frac{\text { vol } .(\text { modified tolerance region })}{\left(\pi \chi_{v, 0.9973}^{2}\right)^{v / 2}|\Sigma|^{1 / 2}[\Gamma(v / 2+1)]^{-1}}
\end{aligned}
$$

where $k(p)$ is $99.7^{\text {th }}$ percentile of $\chi^{2}$ distribution with degrees of freedom, $|\Sigma|$ is the derminant of $\Sigma$, and $\Gamma(\cdot)$ is the gamma function $[1,2,7]$. Estimation of $\mathrm{MC}_{\mathrm{p}}$ can be estimated by estimator from sampling as follows:

$$
\widehat{M} C_{p}=\frac{\text { vol.(modified tolerance region })}{\left(\pi \chi_{v, 0.9973}^{2}\right)^{v / 2}|S|^{1 / 2}[\Gamma(v / 2+1)]^{-1}}
$$

where $|S|$ is the determinant of sample variance-covariance matrix S. For case capabilities indices that use locations with target values T, MPCI is determined by formula:

$$
\mathrm{MC}_{\mathrm{pm}}=\frac{M C_{p}}{D}
$$

where $\mathrm{D}=\left[1+(\mu-\mathrm{T})^{\prime} \Sigma^{-1}(\mu-\mathrm{T})\right]^{1 / 2}$. The value of capability indices of (5) can be estimated by parameters mean vector and covariance matrix from samples.

If the indices value of $\widehat{M} C_{p}>1$, then variation of tolerance of customers demand is greater than the management specification limit, so the production process is said to be capable. Conversely, if the indices value of $\widehat{M} C_{p}<1$ indicates that the variation of demand tolerance is smaller than the management specification limit. Thus, the production process still produces products that are not in accordance with management specifications.

\section{Materials}

\subsection{Multiple customer demand in the inventory control}

The research takes case studies in the apparel industry by using several types of apparel products as quality characteristics focuses on inventory control. Let $D_{\mathrm{ji}}$ is daily customer 
demand, $\mathrm{j}=1,2,3, \cdots, \mathrm{q}$, in lead times $\mathrm{L}_{1}, \mathrm{~L}_{2}, \mathrm{~L}_{3}, \ldots, \mathrm{L}_{\mathrm{k}}$. The customer demand is designed as the number of apparel products requested form consumers on a lead time L. Multiple customer demand as quality characteristics are given in the form of a random variable:

$$
\begin{aligned}
& X_{l}=\left(D_{11}, D_{12}, \cdots, D_{1 L_{1}}, D_{1\left(L_{1}+1\right)}, \ldots, D_{1 L_{k}}\right)^{\prime} \\
& \quad X_{2}=\left(D_{21}, D_{22}, \cdots, D_{2 L_{1}}, D_{2\left(L_{1}+1\right)}, \ldots, D_{2 L_{k}}\right)^{\prime} \\
& \quad(6) \\
& X_{q}=\left(D_{q 1}, D_{q 2}, \cdots, D_{q L_{1}}, D_{q\left(L_{1}+1\right)}, \ldots, D_{q L_{k}}\right)^{\prime}
\end{aligned}
$$

The daily demand variable $\mathrm{D}_{\mathrm{ji}} ; i=1,2,3, \ldots, \mathrm{n} ; j=1,2,3, \ldots, \mathrm{q} ; \mathrm{n}=L_{1}+\cdots+L_{k}$ are a series of random variables that are mutually independent and identically distributed. In this case the lead time $\mathrm{L}$ is a random variable or fixed. Daily demand variables and lead time variables are also assumed to be mutually independent. If the lead time is fixed or deterministic, then the demand variable lead time follows the variable nature of daily demand.

Customer demand is the number of products desired by consumers or market can be probabilistic. Lead time is the time of delay between the start time of the reorder point (the stock level starting the order), while lead time demand is the number of products desired by consumers in the certain period time. Analysis of quality control for daily customer demand in the apparel industry using inventory control system and inventory control model is given by $[11,12]$.

Based on multiple daily customer demand (6), we design $\mathbf{X}=\left(\mathbf{X}_{1}, \mathbf{X}_{2}, \ldots, \mathbf{X}_{\mathrm{q}}\right)$ ' as a q-dimensional quality characteristic with mean vector $\boldsymbol{\mu}$ and covariance matrix $\boldsymbol{\Sigma}$. The mean vector $\boldsymbol{\mu}$ and covariance matrix $\boldsymbol{\Sigma}$ can be estimated by sample mean vector $\overline{\boldsymbol{X}}$ and sample covariance matrix $\boldsymbol{S}$ respectively.

\subsection{Control charts for inventory control}

Before determining the confidence interval for MPCI, we discuss some control charts for inventory control using individual quality characteristics (6). In general, control charts consist of three basic elements: the center line representing the average process, the upper and lower control limit as shown in Fig. 1 [3]. In Fig. 1 shows that, if the customer demand processes under stable conditions, $(1-\alpha) 100 \%$ of the request data will be in the lower and upper control limits.

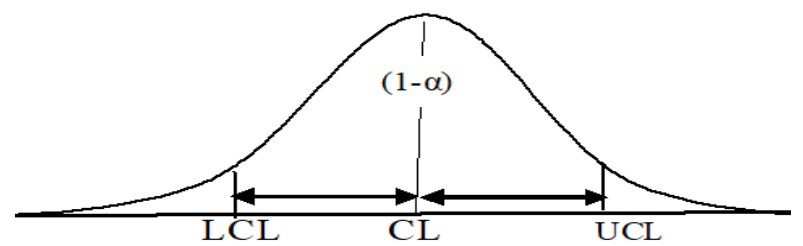

Fig. 1. Upper and lower limit for statistical inventory control

The statistical inventory control uses an average control chart that is used to detect data values between the upper and lower limit. If the all data values are between the upper and lower limits it is said that the process are stable condition, whereas if there is a data value outside the upper or lower limit it is said that the process is unstable. The basic theory for design of inventory control charts is to use a probability of normal distribution to derived the upper and lower control limit, and center line, as given in Fig. 1. Inventory control charts are 
designed with input of daily customer demand D as a stochastic process in a certain lead time L.

\subsection{Control chart for customer demand D}

Let $\mathrm{D}$ is daily customer demand as given in (6). Based on the curve normal distribution in Fig. 1, the center, upper and lower limit for control chart for daily customer demand D can be expressed as the follows:

$$
\begin{aligned}
\mathrm{CL}(\mathrm{D}) & =\mathrm{E}(\mathrm{D})=\mu_{D} \\
\mathrm{UCL}(\mathrm{D}) & =\mathrm{CL}(\mathrm{D})+Z_{\alpha / 2} \sqrt{\operatorname{Var}(D)} \\
& =\mu_{D}+Z_{\alpha / 2} \sigma_{D}^{2}(7) \\
\mathrm{LCL}(\mathrm{D}) & =\mathrm{CL}(\mathrm{D})-Z_{\alpha / 2} \sqrt{\operatorname{Var}(D)} \\
& =\mu_{D}-Z_{\alpha / 2} \sigma_{D}^{2}
\end{aligned}
$$

where CL is the center line, UCL is upper control limit, and LCL is lower control limit, $Z_{\alpha / 2}$ is the normal standard distribution. The parameters value can be determined by value of parameters which can be estimated by average and variance of daily demand D.

\subsection{Control chart for lead time demand $X$}

Let $\boldsymbol{X}$ is lead time demand, that is the sum of number of daily customer demand D for all lead time $\mathrm{L}$ as given in (6) with $\mathrm{D}$ and $\mathrm{L}$ are normal distribution variables. Using the central limit theorem, we can show that the variable oflead time demand $\mathrm{X}$ is normal distribution. As the same way for control chart for customer demand, based on the curve normal distribution in Fig. 1, the center line, upper and lower control limit for lead time demand $X$ can be expressed as the follows:

$$
\begin{aligned}
& \operatorname{CL}(X)= \\
& \begin{aligned}
\operatorname{UCL}(X) & =\mathrm{CL}(X)+\mu_{L} \mu_{D} \\
& =\mu_{L} \mu_{D}+Z_{\alpha / 2} \sqrt{\operatorname{Var}(X)} \\
\operatorname{UCL}(X) & =\operatorname{CL}(X)-Z_{\alpha / 2} \sqrt{\operatorname{Var}(X)} \\
& =\mu_{L} \mu_{D}-Z_{\alpha / 2} \sqrt{\mu_{L} \sigma_{D}^{2}+\sigma_{L}^{2} \mu_{D}^{2}}
\end{aligned}
\end{aligned}
$$

If the lead time $\mathrm{L}$ are fixed, the control chart limit can be expressed as the follows:

$$
\begin{aligned}
& \operatorname{CL}(X)=\mathrm{E}(X)=L \mu_{D} \\
& \begin{aligned}
\operatorname{UCL}(X) & =\mathrm{CL}(X)+Z_{\alpha / 2} \sqrt{\operatorname{Var}(X)} \\
& =L \mu_{D}+Z_{\alpha / 2} \sqrt{L \sigma_{D}^{2}} \\
\operatorname{LCL}(X) & =\operatorname{CL}(X)-Z_{\alpha / 2} \sqrt{\operatorname{Var}(X)} \\
& =L \mu_{D}-Z_{\alpha / 2} \sqrt{L \sigma_{D}^{2}}
\end{aligned}
\end{aligned}
$$




\section{Methods}

Let $\mathbf{X}=\left(\mathbf{X}_{1}, \mathbf{X}_{2}, \ldots, \mathbf{X}_{\mathrm{q}}\right)^{\prime}$ ' have a multivariate normal distribution with mean vector $\boldsymbol{\mu}$ and covariance matrix $\boldsymbol{\Sigma}$. The multivariate of quality characteristics for customer demand control charts is designed using the $T^{2}$ Hotelling control chart as the statistical quality control process to find out whether a process is stable or not. Those control charts are useful for detecting variations in process from being formed from several quality characteristics [3]. The assumptions for analysis of MPCI are the sample data have multivariate normal distribution and stable conditions in the statistical control chart.

The most common procedure for multivariate statistical quality control process is to use a $T^{2}$ Hotellingcontrol chart by looking at the average vector of the process [3], Design $T^{2}$ Hotellingcontrol charts for all sample data using individual $T^{2}$ Hotelling. The individual value $T_{i}^{2}$ in the $T^{2}$ Hotelling for the-i observation can be calculated by formula:

$T_{i}^{2}=\left(x_{j i}-\bar{x}_{j}\right) \boldsymbol{S}^{-1}\left(x_{j i}-\bar{x}_{j}\right)^{\prime} ; i=1,2,3, \ldots, \mathrm{n} ; j=1,2,3, \ldots, \mathrm{q}$.

Furthermore, the limits of control chart used for each observation of $T^{2}$ Hotelling as follows:

$$
\begin{aligned}
\mathrm{UCL} & =\frac{p(n-1)}{n-p} F_{\alpha, p, n-p} \\
\mathrm{UCL} & =0
\end{aligned}
$$

where UCL is Upper Control Limit, LCL is Lower Control Limit and $F_{\alpha, p, n-p}$ is F-distributed with $\mathrm{p}$ and $(\mathrm{n}-\mathrm{p})$ degrees of freedom. An observation of multivariate data is said to be stable if the value of $\mathrm{T}_{\mathrm{i}}^{2}>\mathrm{UCL}$ or if $\mathrm{T}_{\mathrm{i}}^{2}<\mathrm{LCL}$.

The research procedure begins with identifying problems in the apparel industry by taking case studies in the apparel industry. The data analyzed are daily product demand from consumers and lead time from management. In this case using two variables, namely variable of apparel products type I and type II. The observation data is taken from consumer demand in a number of retailers as given in the case study.

The steps of the research include: making the case study in the apparel industry by collecting the data needed in the case study, the design of the multivariate inventory control model adapted to the characteristics of the data obtained in the case study, making the formulas needed in the inventory control model, testing the assumptions of the data used, create multivariate control charts, create multivariate inventory monitoring models according to case studies, determine value of MPCI, and determine interval boundaries for MPCI for case study.

\section{Results and Discussion}

This section provides the results of applying the confidence interval for MPCI in the apparel industry. Case studies take multiple quality characteristics using daily customer demand as given in (6). As the sample data using observational data in the form of daily demand taken for 61 days from 1 October to 30 November 2017 for 2 types of apparel products (type I and type II) from 3 retailer with the number of observation data $n=61$. Data samples were analyzed in the form of daily demand divided into several lead times. Each product has 
different fabric requirements and supplementary materials. The case study discussed a bivariate customer demand in the inventory control. The specification limits for number of customer demand for apparel products of type I is $(100,450)$ and for type II is $(150,800)$. The computational results for parameters of the bivariate customer demand are given in Table 1. The sample mean vector and sample covariance matrix of multivariate custumer demand are:

$$
\begin{aligned}
& \bar{X}^{T}=\left[\begin{array}{ll}
272 & 532
\end{array}\right] \text { and } S=\left[\begin{array}{cc}
2988,255 & 1971,414 \\
1971,414 & 2840,887
\end{array}\right] \\
& |S|=17993986,84
\end{aligned}
$$

The profile of actual daily demand data and UCL and LCL are given in Fig. 2.

Table 1. Bivariate customer demand

\begin{tabular}{llllll}
\hline Type of product & $\mathrm{n}$ & Min & Max & Mean & Var \\
\hline Type I & 61 & 150 & 415 & 272 & 2988,255 \\
Type II & 61 & 346 & 698 & 532 & 2840,887 \\
\hline
\end{tabular}

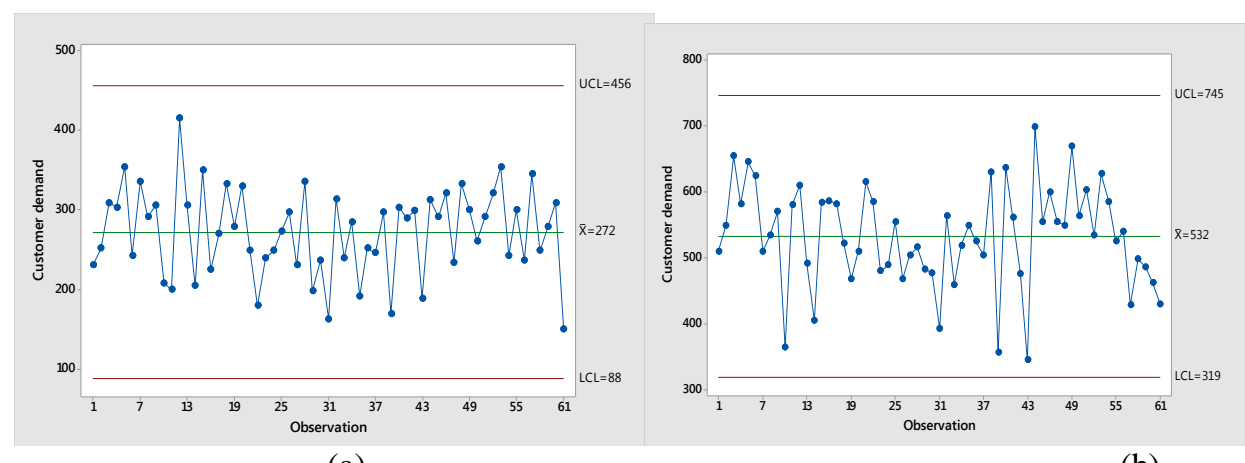

(a)

(b)

Fig. 2. Control chart for customer demand of (a) Product Type I, (b) Product Type II

Based on statistical computing, the variables for two types I and Type II are correlated with a correlation coefficient is 0.472 and probability value is 0.000 . Fig. 2 shown that the variable of customer demand for type I and type II are within the upper bound and lower bound. Therefore, the quality characteristics based on the sample are in the stable condition. Based on the formula (10) and (11), it can be investigated that multiple customer demand in stable condition, as shown in Fig. 3. To prove that the bivariate data of customer demand have a normal distribution is used the Kolmogorov-Smirnov test, which states that actual data of the bivariate data of customer demand is normally distributed for $\alpha=0,05$. 


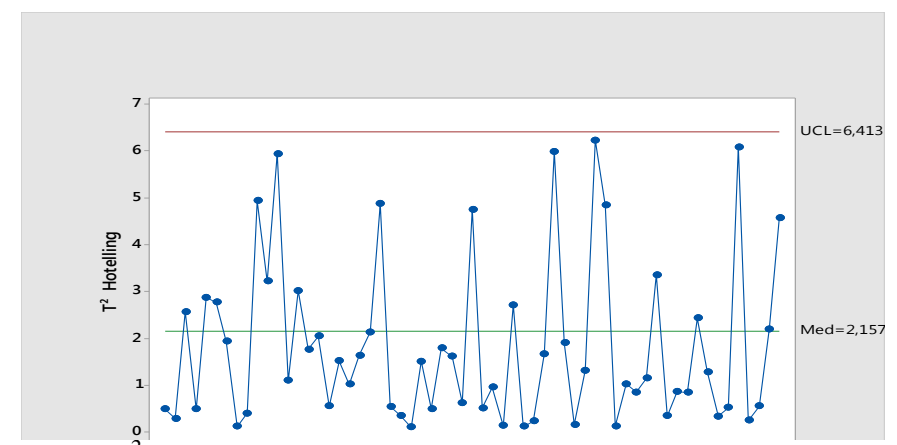

Fig. 3. $T^{2}$ Hotelling control chartfor bivariate customer demand

Furthermore, using the procedure in [1], we get the lower and upper limits for MPCI based on multiple daily demand as given in form (6). Using the formula (4) and (5), $\mathrm{MC}_{\mathrm{p}}$ can be expressed by the formula:

$\widehat{M} C_{p}=\mathrm{MC}_{\mathrm{p}} \times(|\mathrm{S}| /|\Sigma|)^{-1 / 2}$

By using definition 100(1 - $\alpha) \%$ confidence interval for the probability distribution:

$$
\begin{aligned}
\mathrm{P}\left(\mathrm{L} \leq \mathrm{MC}_{\mathrm{p}}\right. & \leq \mathrm{U})=1-\alpha \\
\mathrm{P}\left(\mathrm{L} \leq \mathrm{MC}_{\mathrm{p}}\right. & \leq \mathrm{U})=\mathrm{P}\left\{\mathrm{L} \leq \widehat{M} C_{p} \times(|\mathrm{S}| /|\Sigma|)^{-1 / 2} \leq \mathrm{U}\right\} \\
= & \mathrm{P}\left\{\frac{L}{M C_{p}} \leq \frac{|S|}{|\Sigma|} \leq \frac{U}{M C_{p}}\right\} \\
& =\mathrm{P}\left\{\frac{L}{M C_{p}} \leq \sqrt{\frac{\chi_{n-1}^{2} \times \chi_{n-2}^{2} \times \cdots \times \chi_{n-q}^{2}}{(n-1)^{q}}} \leq \frac{U}{\widehat{M} C_{p}}\right\} \\
& =\mathrm{P}\left\{\frac{L^{2}(n-1)^{q}}{M C_{p}^{2}} \leq \chi_{n-1}^{2} \times \chi_{n-2}^{2} \times \cdots \times \chi_{n-q}^{2} \leq \frac{U^{2}(n-1)^{q}}{\bar{M} C_{p}^{2}}\right\}
\end{aligned}
$$

Let $\mathrm{y}=\chi_{n-1}^{2} \times \chi_{n-2}^{2} \times \cdots \times \chi_{n-q}^{2}$, then based on (11), we have

$$
\int_{L^{2}(n-1)^{q} / M C_{p}^{2}}^{U^{2}(n-1)^{q} / \widehat{M} C_{p}^{2}} f_{y}(y) d y=1-\alpha
$$

Furthermore, based on the characteristic of probability distribution curve, we derived the formula:

$$
F_{Y}^{-1}(\alpha / 2)=L^{2}(n-1)^{q} / \widehat{M} C_{p}^{2} \text { and } F_{Y}^{-1}(1-\alpha / 2)=U^{2}(n-1)^{q} / \widehat{M} C_{p}^{2}
$$

Therefore, a $100(1-\alpha) \%$ confidence interval for $\widehat{M} C_{p}$ can be derived:

$$
\left[\widehat{M} C_{p} \sqrt{\frac{F_{Y}^{-1}(\alpha / 2)}{(n-1)^{q}}}, \widehat{M} C_{p} \sqrt{\frac{F_{Y}^{-1}(1-\alpha / 2)}{(n-1)^{q}}}\right]
$$


For $\mathrm{q}=2$, since the distribution of $\chi_{n-1}^{2} \times \chi_{n-2}^{2}$ is equal to $\frac{\left(\chi_{2 n-4}^{2}\right)^{2}}{4}$. Therefore a $100(1-\alpha) \%$ confidence interval for $\widehat{M} C_{p}$ can be derived:

$$
\left[\widehat{M} C_{p} \sqrt{\frac{\left(\chi_{2 n-4, \alpha / 2}^{2}\right)^{2}}{4 \times(n-1)^{2}}}, \widehat{M} C_{p} \sqrt{\frac{\left(\chi_{2 n-4,1-\alpha / 2}^{2}\right)^{2}}{4 \times(n-1)^{2}}}\right]
$$

Based on the formula (4), for $\mathrm{q}=2, \alpha=0,05$ the estimation of $\mathrm{MC}_{\mathrm{p}}$ can be computed:

$$
\begin{aligned}
& \widehat{M} C_{p}=\frac{\text { vol. }(\text { modified tolerance region })}{\left.\left(\pi \chi_{v, 0.9973}^{2}\right)^{v / 2}|S|\right|^{1 / 2}[\Gamma(v / 2+1)]^{-1}} \\
&=\frac{\pi \times\left[\frac{450-100}{2}\right] \times\left[\frac{800-150}{2}\right]}{\pi \chi_{v, 0.9973}^{2}|S|^{1 / 2}[\Gamma(2)]^{-1}}=1,305
\end{aligned}
$$

Using the formula (13), $95 \%$ confidence interval for $\widehat{M} C_{p}$ is derived as:

$\left[1,305 \sqrt{\frac{\left(\chi_{2 n-4,0,025}^{2}\right)^{2}}{4 \times(61-1)^{2}}}, 1,305 \sqrt{\frac{\left(\chi_{122-4,0,975)^{2}}^{2}\right.}{4 \times(61-1)^{2}}}\right]=[0,974,1,634]$

Based on the computational results above, the estimated capability index MPCI for the performance of the apparel industry is 1,305 , with the confidence interval have the lower bound is 0,974 , and the upper bound is 1,634 .From the results of the calculation of confidence interval for capability index MPCI is $1.305>1$ and the lower limit is $0.974<1$. Therefore, the industry's performance is still not in accordance with management specifications.

\section{Conclusions}

The implementation of specification limits for the capability index in quality control with various statistical quality characteristics is often used in the inventory control. Due to the many characteristics that must be involved in the analysis, the role of the confidence interval for MPCI is very important. The MPCI has been designed to measure process performance based on several quality characteristics, each of which complies with management specification limits. The confidence interval for the MPCI for inventory control is designed for multiple customer demand in the apparel industry. These variables are built from apparel product type I and type II. The application of a confidence interval for inventory control uses the assumption that many customer demands have a normal distribution and stable in the control diagram.

\section{References}

[1] Pearn, W.L., Wang, F.K., Yen, C.H.: Multivariate Capability Indices: Distributional and Inferential Properties, Journal of Applied Statistics. Vol. 34, pp. 941-962 (2007)

[2] Tano I, Vännman K.: Comparing Confidence Intervals for Multivariate Process Capability Indices. Quality and Reliability Engineering International. Vol. 28, pp. 481-495 (2011)

[3] Montgomery, D.C: Introduction to Statistical Quality Control, 6th ed. John Wiley \& Sons, Inc. New Jersey, NJ (2009) 
[4] De-Felipe, D., Benedito, E.: A review of univariate and multivariate process capability indices. The International Journal of Advanced Manufacturing Technology. Vol. 92, pp. 1687-1705 (2017)

[5] Das, N., Dwivedi, P.S.: Multivariate process capability index: a review and some results. Economic Quality Control. Vol. 28, pp. 151-166 (2013)

[6] José, HAR, Alfonso, MMA, Carlos, C.R.J.: Robust Multivariate Process Capability Indices Contemporary Engineering Sciences. Vol. 11, pp. 4139-4146 (2018)

[7] Pan, J.N., CI Li, C.I.: New capability indices for measuring the performance of a multidimensional machining process. Expert Systems with Applications. Vol. 41, pp. 2409-2414 (2014)

[8] Aini, N., Mustafid, Kusumaningrum, R.: Statistical Process Control Systems in Apparel Production. IEEE Xplore: 25 January 2018.

[9] Sabila, A.D., Mustafid, Suryono, S.: Inventory Control System by Using Vendor Managed Inventory (VMI). In E3S Web of Conferences. Vol. 31. pp. 11015 (2018)

[10] Mustafid, Ade Karimariza, S., Jie, F.: Supply chain agility information systems with key factors for fashion industri competitiveness. International Journal of Agile Systems and Management. Vol. 11, pp. 1-22 (2018)

[11] Mustafid, Ispriyanti, D., Sugito, Safitri, D.: Inventory control systems for stochastic lead time demand. E 3S Web of Conferences 73 (2018)

[12] Mustafid, Ispriyanti, D., Sugito, Safitri, D.: Inventory control model using exponential smoothing control chart. Journal of Physics Conference Series 1217 (2019) 